Journal of Universal Computer Science, vol. 27, no. 12 (2021), 1325-1346

submitted: 11/2/2021, accepted: 9/8/2021, appeared: 28/12/2021 CC BY-ND 4.0

\title{
An Integration of Health Monitoring System in Public Transport Using the Semantic Web of Things
}

\author{
Abdelhalim Hadjadj \\ (Computer Science Department, LabSTIC laboratory, University 08 Mai 1945 \\ Guelma, Algeria. \\ https://orcid.org/0000-0003-2270-9830, hadjadji.abdelhalim@univ-guelma.dz) \\ Khaled Halimi \\ (Computer Science Department, LabSTIC laboratory, University 08 Mai 1945 \\ Guelma, Algeria. \\ https://orcid.org/0000-0002-0047-2986, halimi.khaled@univ-guelma.dz)
}

\begin{abstract}
The integration of the Internet of Things (IoT) technology and artificial intelligence has become essential in many aspects of daily life since the expansion of the communications and information field. Healthcare is one area that urgently needs to benefit from these technologies to keep up with the dramatic evolution of communications for contemporary human life. IoT, through wearable devices, provides real-time data related to the measurement of a person's vital signs of health. However, for this data to become more relevant and valuable, it needs to be linked to other domains. Public transport is a domain related to the daily activity of people who take advantage of the IoT to provide exemplary transport services whose quality of service can greatly affect people's health. The integration of these two domains offers many benefits, especially when providing services adapted to passengers' health status, making them safer and healthier. This paper proposes an approach based on an IoT architecture using Semantic Web technologies; it aims to integrate health monitoring in public transport, provide passengers with quality transport services, and ensure continuous health monitoring. The use of Semantic Web technologies overcomes the lack of interoperability due to the heterogeneity of data collected by different devices and generated by two different domains. An experimental study was conducted, and the proposed approach's results were compared with those obtained by the evaluation of a physician. The results show that the approach is effective and should allow passengers to benefit from appropriate transport services that better match their health status.
\end{abstract}

Keywords: Internet of Things, Semantic Web, knowledge-based ontology, SWRL, interoperability, health care, public transport

Categories: B.4.2, L.1.4, I.2.4, D.2.12, K.4.2

DOI: $10.3897 /$ jucs.76983

\section{Introduction}

In recent years, the world has witnessed the spread of many new diseases, some of which are deadly and others dangerous. Moreover, these diseases are affecting more and more people every day. Health surveillance is one of the best solutions to save a human life if appropriate actions are taken at the right time. However, most medical interventions are carried out in health facilities such as hospitals, limiting their effectiveness. Some patients need real-time monitoring, especially for diseases that 
occur suddenly, such as heart failure and Covid-19. The performance of health systems can be improved by using the large amount of data collected daily.

Indeed, it is very easy today to collect data using various devices: sensors, scanners, smartphones, biometric devices, imaging devices, cameras, video surveillance, etc. The majority of these tools are driven by computers and often connected to computer networks, storing massive data that could be used to improve health. Most of these tools are computer-driven and often connected to computer networks, storing massive data that could be processed and manipulated. The Internet of Things (IoT) is a new paradigm that provides intelligence to physical objects by equipping them with sensors, actuators and frequency identification tags (RFID) to produce and exchange data and offer new services. IoT provides a great opportunity to make better decisions, increase efficiency and improve the quality of services.

The goal of the health care field is to provide high-quality healthcare services to keep people safe and alive. To improve wellbeing, IoT helps health care services by providing a large amount of data about a person's health to make the best decisions at the right time. Wireless Body Area Network (BAN) technology and wearable devices allow data to be collected and sent in real-time to hospitals or physicians, making health monitoring possible and easy. Public transport can play a crucial role in improving the performance of health systems, as millions of people use it daily, even for long hours. These vehicles can become early diagnostic devices for passengers. To do this, they are equipped with advanced sensors and devices that allow them to communicate with passenger's wearable devices or collect real-time information about their health. This information is then transmitted to transport agencies or hospitals for analysis and appropriate decisions.

The increasing diffusion of IoT in various fields drives manufacturers to create their own IoT infrastructure, specific devices, communication protocols and network connectivity methods. All these issues create communication difficulties in reading different data formats, specific contexts, and data must be exchanged vertically, a phenomenon known as "vertical silos". Interoperability is one of the challenges of the IoT. It is concerned with the integration of heterogeneous devices and the handling of data formats. It helps to reduce the fragmentation of the IoT domain and ensures the provision of services to all customers [Bröring et al., 2017].

Semantic interoperability is one of the key levels of interoperability. It refers to exchanging data in a meaningful way to be interpreted automatically by machines [Ganzha et al., 2017]. It ensures that data is exchanged in a meaningful and consistent way to be interpreted automatically and correctly across multiple platforms and between different domains. Researchers use the Semantic Web to achieve semantic interoperability. Semantic Web is considered as the next generation of the web that enables the semantics of information and services. The Semantic Web is based on a set of standards: RDF, RDF Schema, OWL to represent data, and SPARQL to query that data. This integration of the IoT with the Semantic Web Semantic Web is called the Semantic Web of Things (SWoT).

This paper proposes an approach based on the IoT architecture that uses the Semantic Web to achieve semantic interoperability between two domains (public transport and healthcare). We propose an ontology that coherently represents data collected from heterogeneous resources, a set of SWRL rules to enrich the ontology by inferring new hidden relationships, and a reasoning engine to infer new knowledge about passengers and their health. This approach aims to safeguard the health of 
passengers by providing them with quality transport services, adapting to their health status and applying real-time semantic processing on the heterogeneous data collected.

The rest of this paper is organised as follows. Section 2 gives a brief overview of the IoT and its evolution towards a SWoT, the challenges of interoperability and ontology in the IoT domain, IoT in health care and public transport. In section 3 , we present related work. Section 4 describes the general architecture of the proposed approach, while Section 5 presents the conceptual architecture with its acquisition layer, application layer and semantic layer. Section 6 presents a scenario of a passenger using public transport through the proposed system. Section 7 presents an implementation with an evaluation and experimentation. Finally, a conclusion with future work is presented in section 8 .

\section{Background}

This section provides a brief overview of the Internet of Things, the Semantic Web and the Semantic Web of Things, public transport and healthcare.

\subsection{Internet of Things}

The Internet of Things (IoT) is a new paradigm invented by Kevin Ashton [Ashton et al., 2009]. It turns things into smart objects by equipping them with electronics, software and sensors that allow them to connect to the internet to collect and exchange data anytime, anywhere and with anyone. IoT has attracted many companies to increase their revenues and dominate the market with several distinguished products by their own devices, architecture and use of personal protocols and platforms. Therefore, the exchange of data in a vertical way called "vertical silos", where standardisation is absent, is due to the lack of interoperability in the IoT domain.

Interoperability is seen as a central challenge for the IoT. It enables the integration of new devices with any platform; it aims to enable rapid adoption and provide users with seamless access to different IoT services. Lack of interoperability prevents the IoT from reaching its full potential value [Manyika et al., 2015]. Researchers classify IoT interoperability at different levels, some have different names, and some have more levels than others. However, all agree on three crucial levels [Noura et al., 2019]: technical, syntactic and semantic interoperability.

\subsection{From IoT to SWoT}

The web of things is an evolution of the IoT that addresses the fragmentation of the IoT to enable interoperability between devices [Faheem et al., 2019]. As the primary means of communication on the internet, the web provides an application layer that simplifies the IoT and enables data sharing across the globe. Despite its benefits, there remains a lack of common understanding of the diversity of things provided by the Semantic Web. The Semantic Web [Hitzler, 2021] is the next generation of the web, making it more machine-readable. It offers a promising solution for a formal and shareable representation of knowledge. It is based on a set of standards such as the URI, a string for identifying resources. $\mathrm{RDF}^{1}$ is a triple declaration (subject, predicate and object)

${ }^{[1]} \mathrm{RDF}$ (Resources description framework): https://www.w3.org/RDF/ 
that expresses data and includes XML-based resource metadata. The RDF schema ${ }^{2}$ and OWL ${ }^{3}$ allow for the greater expressiveness needed to build ontologies, and SPARQL allows for querying the knowledge base.

Ontology is "a formal, explicit specification of a shared conceptualization"” [Studer et al. 1998]. It is considered the backbone of the Semantic Web; it describes a domain and specifies its terms and relationships. It provides robust solutions to heterogeneity problems by dynamically using shared vocabularies. Continuous improvement of the Avancha et al. ontology [Avancha et al., 2004], considered the first ontology in the IoT domain, has led to significant steps to develop the Semantic Sensor Network (SSN) ontology [Compton et al., 2012] which describes the core concepts common to all IoT applications. The popular OneM2M platform provided OneM2M [Alaya et al., 2015] as a minimal ontology that requires more concepts to represent a device and its functionality. ETSI (European Telecommunications Standards Institute) published the Smart Appliances Reference (SAREF) ontology for energy efficiency relevant devices and then became a standard ontology that can provide a shared model for consensus mapping of existing assets. The SAREF ontology provides building blocks that allow the reuse of different ontology parts according to specific requirements [Daniele et al., 2015]. Like the SSN ontology, SAREF has expanded to cover other domains such as SAREF4Wear for a wearable device, etc. SWoT integrates Semantic Web technologies with the IoT [Scioscia and Ruta, 2009]. It improves people's lives by providing large-scale interoperability to the domains that adopt it, allowing the description, sharing and reuse of collected data.

\subsection{IoT to Improve People's Lives}

Connecting devices to the internet will allow them to communicate with each other and transfer information, which will help people to do their work better and faster and increase the efficiency and quality of services. It will also provide people with a huge amount of information to be used in research and studies to develop and improve performance in all areas of life. This work highlights how the IoT can be used in the health and public transport sectors because of its great importance for improving the daily life of citizens.

\subsection{Health care}

The medical sector has benefited from digital transformation and modern technologies. It is expected to rely increasingly on the IoT in the coming years [Kelly et al., 2020], with wearable medical devices, mobile health applications, and services helping shape innovative features in how healthcare services are delivered. IoT technologies in health care have helped to improve the daily work in hospitals. Medical data is collected mainly through the use of wearable devices. The availability and sharing of data have been instrumental in maintaining patient safety and health, improving the way physicians deliver care, and increasing patient engagement and satisfaction by allowing them to spend more time interacting with their physicians.

A wearable device is any device capable of providing real-time sensing information about its wearer through a set of sensors; it can be worn as an accessory or embedded

${ }^{[2]}$ RDF schema https://www.w3.org/TR/rdf-schema/

${ }^{[3]}$ web ontology language (owl): https://www.w3.org/OWL/ 
into clothing. According to Statista ${ }^{4}$, the number of wearable devices is growing by about 325 million between 2016 and 2019. It is expected to exceed 1 billion by 2022, indicating the increase in their popularity and the growing public awareness of the importance of their relevant data, especially for their health. In addition, the interest of physicians and hospitals in using this technology to diagnose patients gives patients more confidence to wear it and follow the instructions sent through it.

The wearable device provides valuable information (vital signs) such as time, activity duration, sleep status, physical activity, etc. [Takei et al., 2015]. It can also identify health problems and monitor heart rate, pulse, temperature and sugar levels in the body and digestive system. These connected devices can help diagnose health problems, take vital data from the body throughout the day and transfer it wirelessly to physician's devices such as computers and smartphones for analysis and appropriate medical action.

\subsection{Public Transport}

Public transport is a vital economic sector in every country in the world. It allows passengers to move from one place to another for work, study, care, leisure, etc. IoT in public transport consists of sensors that allow agencies to collect data and actuators to trigger certain events in the means of transport and to exchange real-time information about passengers within these means. Passenger health monitoring can be leveraged to improve the health care performance of the health care system. It can provide hospitals or health care centres with accurate, real-time information on the health status of passengers, allowing for accurate diagnosis and reducing pressure on hospitals by only treating people in real-life situations. This is what we experienced and are still experiencing in the period of the Covid 19 pandemic, where most hospitals became unable to handle the huge number of people infected with the coronavirus. Unfortunately, after a period of diagnosis and analysis, some patients diagnosed with non-serious conditions are being discharged from the hospital. At the same time, we have lost human lives requiring urgent treatment because there are not enough resuscitation beds. This scenario could be avoided by integrating the monitoring of a passenger's health status by IoT technology inside the vehicles to get an accurate picture of public health.

Public transport and health care systems can leverage a SWoT to manage the heterogeneity of data collection and exchange it in a meaningful way. Data converted into meaningful context allows agencies to increase profits by providing timely services, comfortable travel and adaptability to passenger health requirements. Depending on the health status of the passengers, the provision of adaptable services is seen as a dual objective: to add benefits to the sector and ensure continuous monitoring of patients' health. This can be achieved by using the wearable devices that people carry widely today.

\footnotetext{
${ }^{[4]}$ https://www.statista.com/statistics/487291/global-connected-wearable-devices
} 


\section{$3 \quad$ Related Work}

Many researchers have looked at health monitoring systems that are related to the process of collecting data from sensing wearable devices. An example of this work, Rhayem et al. in [Rhayem et al., 2017] highlighted a semantic representation of both connected medical objects and their data by proposing a health IoT ontology for patient monitoring. The work is based on the analysis of the obtained vital signs and semantic rule reasoning to help decision-makers. Reda et al. in [Reda et al. 2018] also proposed an ontology representing health data from heterogeneous IoT sources and allowing automatic reasoning by inference engines to facilitate interoperability and integration of health data. El-Sappagh et al. in [El-Sappagh et al., 2019] propose a comprehensive mobile health framework with an integrated clinical decision support system (CDSS) to achieve semantic interoperability with different data sources from clinical systems. It is cloud-based and can be used to manage type 1 diabetes mellitus. The authors proposed the FASTO ontology to collect, formalise, integrate and analyse patient data. This work represents the domain data that they defined the ontology from scratch. However, to reduce the development cost, Moreira et al. reused a standard SAREF ontology to define its extension, namely SAREF4health. It allows semantic interoperability of IoT solutions that have to deal with frequency-based time series [Moreira et al., 2020]. To prove the performance of their ontology, the authors represented a time series of real-time ECG sensor measurements to detect accidents with truck drivers.

Other studies have focused on the integration of IoT technologies to improve the public transport domain. Most of them aim to facilitate the process of data collection and communication between different resources. For example, [Ryu et al., 2020] present a WiFi sensing system to estimate passengers' waiting time at bus stops using their smartphone MAC address and [Frez et al., 2019] present a method to support the decision making of transport networks based on crowdsourced data. Using the Semantic Web, Houda et al. in [Houda et al., 2010] have developed a public transport ontology that considers several concepts related to travel planning. It allows passengers to choose the best route to get from one place to another. The system could respond to simple user queries. [Bermejo et al., 2014] also proposed decentralising decision-making by integrating an ontology inside each vehicle to give them reasoning capabilities in case of an emergency. [Benvenuti et al., 2017] proposed an approach to facilitate the public transport monitoring system based on the integration of ontological key performance indicators (KPIOnto) and transmodel ontologies (the European reference data model for public transport information systems). SPARQL can query the proposed ontology to extract relevant information, but the proposed method suffers from the different structural nature of the two approaches it integrates.

Based on the research work presented above, we conducted a comparative study summarised in Table 1. This study shows that most approaches use semantic web technologies to represent and model the data created and exchanged by different IoT systems. However, most researchers have developed their ontology from scratch and have not taken advantage of using existing standards that can help represent domain knowledge appropriately. Some of the works are cloud-based systems; the researchers have not proposed alternative architectures that could reduce decision-making latency and use different existing data resources. The work presented concerns human health 
monitoring or public transport monitoring. We believe that the fusion of these two domains can bring more benefits to both domains and will inevitably improve the quality of life of human beings.

\begin{tabular}{|l|l|l|l|l|l|l|l|l|l|}
\hline \multicolumn{1}{|c|}{ Work } & \multicolumn{3}{c|}{$\begin{array}{c}\text { Semantic Web } \\
\text { technologies }\end{array}$} & \multicolumn{2}{l|}{ Domains } & \multicolumn{2}{|l|}{ IoT } \\
Architecture
\end{tabular}

Table 1: Qualitative comparison of public transport, health monitoring, IoT-based cross-domain work and the Semantic Web

After analysing the literature in the fields of SWoT, as well as the state of the practice in the application of Semantic Web and IoT in health care and public transport, a description of a proposed system (passenger transport monitoring system) that combines the technologies mentioned above is presented in the following sections.

\section{Model for Integrating Health Monitoring in Public Transport}

A system that integrates public transport with health monitoring contains several objects that can generate and exchange data and then process and provide services to end-users through applications. Most applications for the health monitoring or public transport domains are cloud-based, but others intend to decentralise this model to handle distributed data, including data generated by the IoT. Similar to Mohan's architecture that classifies computational devices according to their capabilities [Mohan et al., 2016]. Figure 1 shows a model consisting of four layers: the devices layer, the edge layer, the fog layer and the cloud layer. At the lowest level, we can find all the objects for collecting data or taking measurements. This layer consists of IoT devices which can be sensors, actuators or wearable sensing devices. Sensors installed in vehicles or bus stops collect data about the environment, such as location, number of passengers, vehicle status, etc. Actuators allow taking actions. Actuators allow actions to be taken, such as opening a vehicle door or triggering an alarm, etc. Wearable devices allow health monitoring through a set of sensors working in synchronisation. They are characterised by their low cost, ease of use and come in the form of a smartwatch, glasses, etc. 


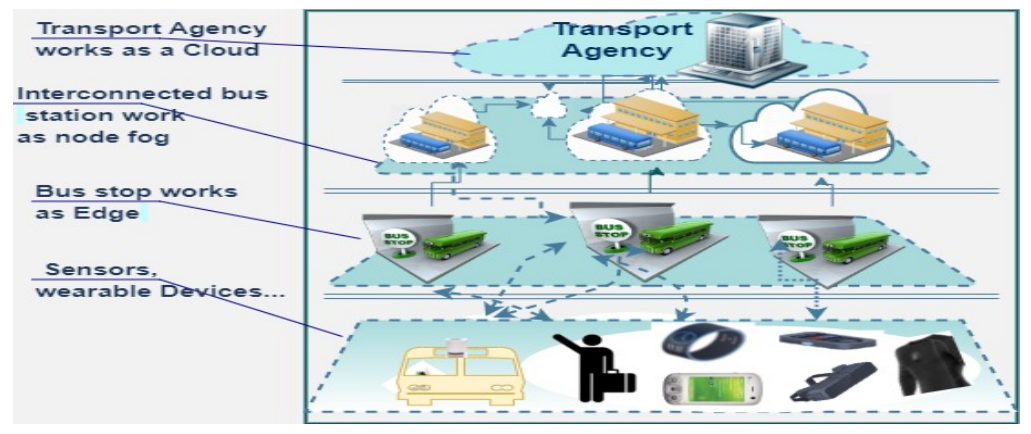

Figure 1: Architecture of the patient transport monitoring system model

The most important part of the architecture is the second layer, which presents the Smart Bus Stop (SBS). The smart bus stop works as an edge to improve the management, local storage, and real-time data processing generated by connected devices associated with public transport and health monitoring. The SBS can identify and connect a passenger through the MAC address of his/her wearable devices or smartphones. It receives data from wearable sensors and vehicles using wireless communication protocols. It can get more information from other bus stops or highlevel transport managers to aggregate the collected data. The smart bus stop calculates data close to its sources. It analyses and processes the data and sends the results to passengers, vehicles or anyone interested in the results. It helps to make quick decisions because the smart bus stop is very close to the connected devices.

Smart bus stops accept to communicate with their neighbours; however, they cannot cover public transport in large areas such as a small city, which requires more processing and storage capacity. In the third layer, the small bus station is considered a fog node to enable computation, networking, and data management data collected by many connected devices. A fog node enables low latency and reduces bandwidth by not sending every bit of information to the cloud. It bridges the gap between the transport agency and smart bus stops. It also provides temporary storage of passenger health data released by the smart bus stop to avoid repeating the process.

In the top layer of the architecture, the transport agency considers a cloud to perform complex calculations, long-term storage of processed data on vehicles, routes, smart bus stops and passenger medical profiles. Transport agencies can exchange passenger data with a hospital to check or detect a new disease, such as a pandemic, by observing a global change in patient data in a region or area. The transport agency allows the data collected to improve the quality of the service and facilitate its management. For example, the time and place of passengers at a bus stop allows the system to calculate the waiting time and reduce it.

This architecture is characterised by the possibility of adding other sources like wearable devices, smart bus stops and bus stations. It also reduces the load on the network because the processing is done on the devices that generate the data. In addition, when one server fails, it does not affect the other servers and prevents the system from coming to a complete stop. The architecture reduces the cost of setting up the infrastructure by providing tasks with adequate resources. In addition, it will be 
more useful for the public transport sector as it allows them to take full advantage of the different technologies offered by this architecture.

\section{Conceptual Architecture}

In the following, we present the conceptual architecture of the system that allows the smart bus stop, bus station and transport agency to monitor passenger health and generate effective recommendations.

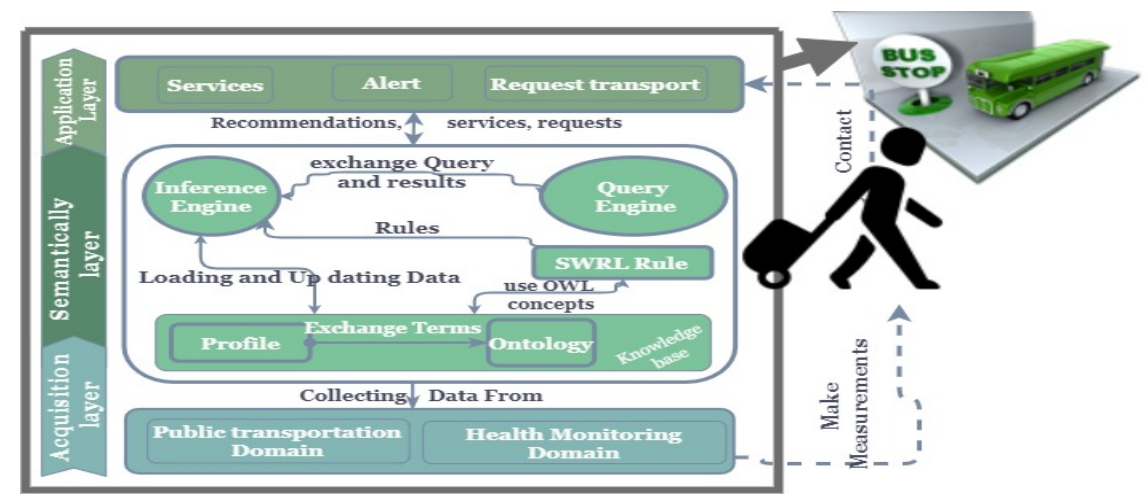

Figure 2: The conceptual model of the proposed system

The proposed conceptual architecture, shown in Figure 2, consists of the acquisition layer, the semantic layer and the application layer. A single communication layer can replace the acquisition layer and the application layer with a bus station or transport agency architecture to connect to each other and smart bus stops.

\subsection{Acquisition Data Layer}

The system acquires data from various sources that are used to monitor passenger vital signs and transport data. The main source of data is the sensors embedded in wearable devices and vehicles. The sensors take real-time measurements and transmit them over the network to the smart bus stops to collect distributed heterogeneous data for analysis and interpretation.

\subsection{Semantic Layer}

The semantic layer is the core of the system. It allows the management of heterogeneous data generated by IoT devices; it consists of the following modules:

\subsubsection{Knowledge Base}

The knowledge base allows information to be described, organised and shared to facilitate its analysis and reuse. It allows representing data acquired from sensors by 
defining concepts and relationships between these concepts (ontology). In addition, it accepts the addition of new information from medical profiles (RDF files).

\subsubsection{Ontological module}

The ontology is a formal model that represents all concepts related to health monitoring and public transport integration and describes their relationship. The development of the ontology representing the integration of health monitoring systems with public transport data went through three stages; it combines three scenarios from the nine scenarios of the Neon methodology [Suárez-Figueroa et al. 2015]. First, we followed Scenario 1, which presents how to develop a new ontology from scratch in case of the absence of an ontology that does not satisfy the domain specification, which is the case of public transport using IoT and supporting the concepts of bus stops, bus stations and transport agencies. In the second step, to take advantage of existing standard ontologies that reduce cost, we followed Scenario 3 by reusing the SAREF4Wear ontology for wearable devices and health monitoring support. In the third step, we followed Scenario 5 , where we reused the two previous ontologies, merging them to design a new ontology called passenger transport monitoring (PTM ontology).

\subsection{Public Transportation Ontology}

IoT data can be collected and transmitted from different public transport sources characterised by their heterogeneity and lack of formality, leading to difficulty in sharing meaning and reuse. The ontology allows us to annotate and describe public transport resources related to IoT systems such as smart bus stops, vehicles, bus stations, etc. As a common language, the ontology meets the requirement of the domain by providing answers to basic questions and other questions related to public transport: What are public transport resources? What are the services provided by public transport? How to communicate public transport resources?

We can define and represent concepts as a class or sub-class by answering these and other questions. In the following, we present the main concepts:

Person: represents the public transport user. The class has other subclasses such as Passenger, Driver and associated with the requestTransport property with the SmartBusStop concept and beniftService with the Vehicles concept.

TransportAgency: represents the agency responsible for managing public transport resources and services. It includes the subclass of small bus stations that manage public transport in a small area or in a few streets.

SmartBusStop: represents a location along a route where vehicles stop to pick up or drop off passengers. This concept is linked to many properties. For example: the provideService property to the Person range concept.

BusStation: represents a small agency for managing public transport. It is associated with the vehicle and the SmartBusStop through manage property.

Vehicle: describes the means of transport. It has several sub-classes, for example, the Type-of-Vehicle, to represent the mode of transport.

To facilitate the merging of this ontology with the standard SAREF ontology, we defined the ontology terms that express concepts of public transport domains as similar to the terms used in the SAREF ontology, such as: 
Device: Physical objects that perform a task in public transport. It includes subclasses of sensors and actuators. They are linked to the vehicle concept through the equippedIn property.

Measurement: represents an operation performed by devices on a property to obtain its value. It uses the mackeMeasurementBy object property to associate with the concept of sensor range and uses the data properties: hasTimeDate, hasValue, etc.

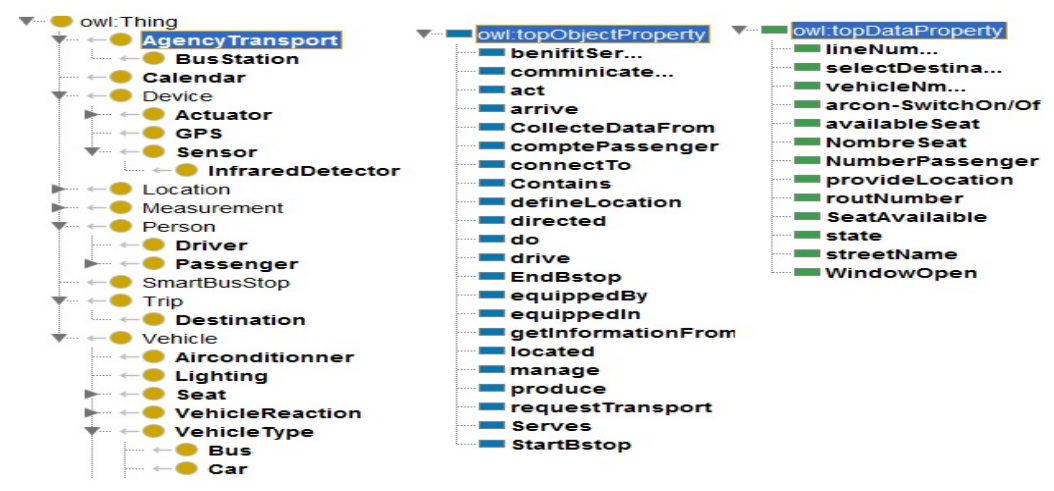

Figure 3: The proposed ontology

The proposed ontology presented in Figure 3 is defined in terms of classes, properties and individuals (class members). It allows sharing a common understanding of heterogeneous data in the public transport domain using IoT, facilitating knowledge reuse and analysis that enables seamless vehicle management and providing passengers with appropriate information and services in the shortest time and at the lowest cost.

\subsection{Wearable Device for Health Monitoring Ontology}

To reduce the costs associated with ontology development by avoiding designing the ontology from scratch. Furthermore, to improve the quality of reused ontologies and ensure that ontology features such as low complexity, lightness, reusability, extension support, and alignment are respected in our ontology, we chose the standard ontology SAREF extension SAREF4wear. This is an ontology also designed by ETSI to represent the wearable devices domain.

To represent the domain knowledge of wearables in health monitoring, some classes of this ontology and their subsets are reused, such as Devices with their subset Sensors, Actuator, and Biometric -property. The classes Measurement, Feature-ofinterest Property, and unit-of-measure are also selected. In addition to the object properties that define the relationship between these classes such as measured-By, consist-Of, make-measurement, relate-To-Property, is-Measured-In, and data type such as has-Value, Has-Timestamp.

\subsection{Passenger Transport Monitoring Ontology}

We performed some operations to develop the passenger transport monitoring (PTM) ontology: reuse of the two previous ontologies, merging and inserting new classes. Thanks to the development of the public transport ontology, which is approximate and 
similar to the SAREF ontology in its structure, the terms share some concepts, which facilitated the merger.

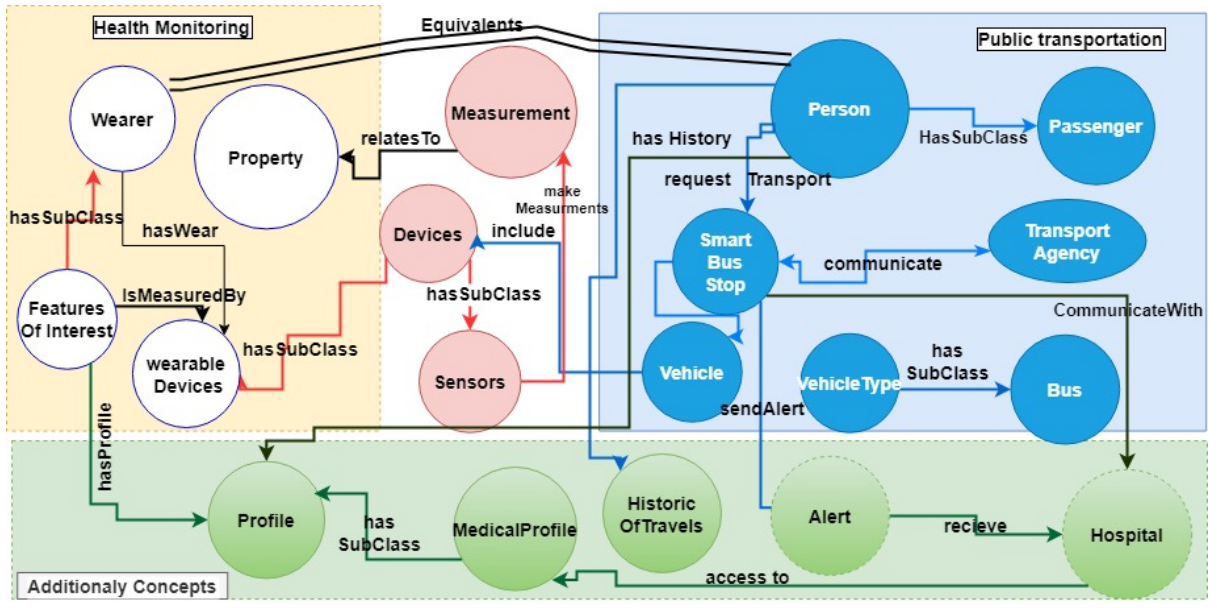

Figure 4: Ontological scheme for monitoring passenger transport

The ontology described in Figure 4 contains some classes from the two previous ontologies and their relationship and data type. To deal with the problem of the existence of some classes that have the same terms in both ontologies, such as devices, measurement and unit of measurement. We merged the classes that share the same terms into one class with all their subclasses and their data relations and properties. We also defined new relationships to link classes from different previous ontologies, such as the Person class from the Public Transport ontology linked by equivalentTo to the Wearer class from the Wearable Health Monitoring Devices ontology.

To obtain a better representation of the knowledge of both domains, we enrich the ontology with new concepts. For example, the concept of medical profile contains information about passengers with their subsets of classes, such as age, medication, medical history, etc. We also enrich the ontology with new relationships that represent the interaction between these concepts such as hasMedicalProfile, IsSmoking,etc.

\subsubsection{Profile Module}

The profile module is an RDF file that contains medical profiles, personal information, history, travel history, etc. The medical profile is an essential part that includes information about the passenger's health status, such as previous illnesses and treatments. The personal profile refers to general passenger information, such as age. The medical profile refers to information on the history of diagnoses and some exceptional services provided to passengers during his/her past travels. The file can be stored in a wearable device such as a smartwatch or smartphone and can be shared and accessed by the physician for consultation or to add instructions to the patient. 


\subsubsection{Inferring new Knowledge about the Passenger}

The knowledge base contains an ontology that plays a crucial role in representing the data, but it cannot express all relationships, especially hidden ones. The inference module based on the Semantic Web Rule Language (SWRL) allows us to infer new knowledge about passenger patients.

In the following, we present some rules written by SWRL that can be expressed in terms of OWL concepts to improve the passenger transport monitoring process.

- R1: Person $(? p)^{\wedge}$ SmartBusStop(?SBS $)^{\wedge}$ requestTansport(?p, ?SBS) $\rightarrow$ Passenger(?p).

If the person $P$ asks for a means of transport, then s/he considered as a passenger.

- R2: Passenger $(? P)^{\wedge}$ SmartBusStop $(? S B S) \wedge$ requestTransport(?P, ?SBS)^ Vehicle(?V)^ connecte(?SBS, ?V)^ InfraredDetector(?I)^ containsDevice(?V, ?I)^ infraredDetectorValue(?I, ?vl)^ swrlb:lessThan(?vl, 30) $\rightarrow$ has Cpacity $(? V \text {, true })^{\wedge}$ provideServices(?SBS, ?P) $\wedge^{\wedge}$ benifitService $(? P, ? V)$.

If the smart bus stop $S B S$ receives transportation requests and the bus capacity is accepted to add more passengers, then provide them with transport service.

- R3: Passenger(?P) ${ }^{\wedge}$ SmartBusStop $(? S B S)^{\wedge}$ requestTransport(?P, ?SBS)^ WearableDevices $(? W)^{\wedge}$ collectDataFrom $(? S B S, ? W)^{\wedge}$ featuresIsMeasuredBy $(? P, ? W)^{\wedge}$ Sensor $(? S) \wedge$ consist $O f(? W, ? S)^{\wedge}$ Measurement $(? M)^{\wedge}$ makeMeasurement $(? S, ? M) \wedge$ Property $(? P r) \wedge$ propertyName(?Pr, "SPO2")^ relatesToProperty(?M, ?Pr)^ has Value(?M, $? v l)^{\wedge}$ swrlb:greaterThan(?vl, 95) $\rightarrow$ provideServices(?SBS, ?P).

If passenger $P$ uses their wearable device to make the measurement and its values are normal, then the smart bus stop SBS offers regular service.

- R4:propertyName(?Pr,"BodyTemperature")^ hasValue(?M,?v)^

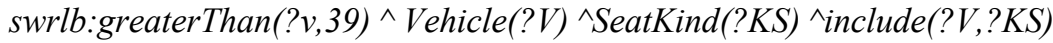
$\wedge$ Airconditionner $(? A c)^{\wedge}$ include $(? V, ? K S)^{\wedge}$ connecte $(? S B S, ? V)^{\wedge} \rightarrow$ provideServices $(? S B S, ? P)^{\wedge}$ VehicleType(?V,"Bus") ^ArcSwitch(?Ac, true). If the measurements of passenger $P$ are abnormal, then the smart bus stop $S B S$ offers a specific service, for example, a seat with a switch to start the air-conditioner.

- R5: propertyName(?Pr,"BloodPressure") ^ relatesToProperty $(? M, ? P r) \wedge$ hasValue(?M, ?v $)^{\wedge}$ swrlb:greaterThan $(? v, 180)^{\wedge}$ Vehicle(?V)^

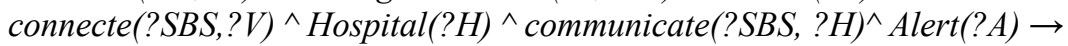
provideServices(?SBS, ?P)^ VehicleType(?V,"emergency car") ${ }^{\wedge}$ sendAlert(?A, ?H).

If the measurements of passenger $P$ show that his/her health is in danger, the smart bus stop $(S B S)$ provides an emergency car and immediately alerts the hospital.

\subsubsection{The Inference Engine and Reasoning Engine}

Semantic reasoning is used to exploit the knowledge base, which contains the ontology, SWRL rules and medical profile, to create new relationships that can extend the ontology, ensure consistency, and infer meaningful information from the semantic sensor data. To semantically interpret the IoT data, we can use one of the available reasoners like Pellet, Hermit, etc. 


\subsubsection{Query Engine}

The query engine used to evaluate and enrich the data through queries facilitates retrieving desired information from the ontology. It is based on the common query language SPARQL (Simple Protocol and RDF Query Language) to create queries that explore semantic descriptions and discover IoT resources. Some examples are presented in the following:

Q1: determine the number of passengers' requesting transport:

Select count (?passenger) \{

where ?smartBusStation TPM :requestTransportation ?passenger\}

Q2: the passengers have a disease and benefit a transport:

SELECT ?Passenger ?disease ?Vehicle

WHERE \{ ?PassengerTPM:requestTransport ?SmartBusStop .

?PassengerTPM:benifitService ?Vehicle.?PassengerTPM:hasDisease ?Disease .

?DiseaseTPM:diseaseName ?disease\}.

\subsection{Application and Services Layer}

Provide users with an interface that helps them request a service offered by the system or exchange data. It helps passengers to obtain the appropriate service according to their health condition. This layer allows passengers to be notified of diagnostic results and offered services. The system enables communication and addresses the challenge of knowledge exchange between heterogeneous IoT devices and between two domains and inferring new knowledge using the Semantic Web. However, it exploits additional data that increases its processing time, which it manages to control through the proposed architecture.

\section{Passenger Scenario with the Proposed System}

This section explains how the approach works through this case scenario. Suppose a person arrives at a bus stop to travel and has a wearable device that records his/her vital signs and health measurements. The smart bus stop detects the MAC address of his/her wearable device or his/her smartphone to contact him/her and send a message asked $\mathrm{him} /$ her to enter his/her destination. When the person assigns a destination, the bus stop considers him/her as a passenger <Ht : Person Ht: requestTransportHt: SmartBusStop $>$. The smart bus stop to meet the transport demand checks the status of nearby vehicles < Ht: smart bus stop Ht: collect-Data-From Ht: vehicle $>$ to know the number of available seats. It starts receiving and collecting data transmitted by the passenger's wearable devices indicating his/her health status $<\mathrm{Ht}$ : wearable $\mathrm{Ht}$ : makeMeasurementHt: passenger $>$, his/her medical profile which is stored in his/her wearable device, and his/her travels history. It is possible that the medical profile is stored in the wearable device, in the previously used smart bus stop (edge), or in the bus station (fog) that manages the smart bus stops. If the bus station does not contain passenger data, it connects to the transport agency (cloud) to retrieve it.

The transport agency provides small bus stations, and smart bus stops with the required information and passenger travel history if this data does not exist at their level. It provides all management and service information. It can also connect to the hospital to exchange health status information. 
After the smart bus stop gets the required information, it uses semantic relations, SWRL rules, inference engine and SPARQL queries to represent the content of these resources. It interprets it semantically to provide suitable recommendations and services < Ht: smart-bus-stop Ht: provide-Service Ht: Passenger> and/or even alerts in case of a dangerous situation.

The Smart Bus Stop (SBS) offers passengers a range of services and recommendations based on their health status. The passenger receives a message informing him/her of his/her health status, the transport services suitable for him/her, and reminding him/her to get his/her medication or consult a physician.

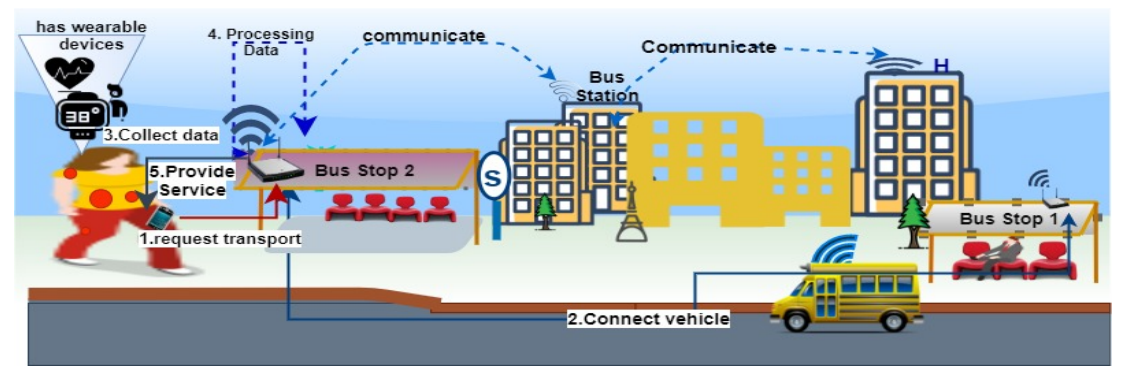

Figure 5: Passenger scenario with the proposed system

For example, Maria in Figure 5 is a passenger who wears a smartwatch; it can take measurements and exchange health data with her smartphone. She asked to be transported by the carrier; she logged in at the smart bus stop and entered her destination. The measurements and medical profile data were transferred as follows: Blood sugar $=160 \mathrm{mg} / \mathrm{dl}$, Blood Pressure $=138 \mathrm{mmHg}(\mathrm{D})$, Heart Beat $=105$, Body Temperature $=38^{\circ} \mathrm{C}$, and personal information: Age: 48 years, Gender: female. After collecting the data on the vehicle's condition, the smart bus stop system loads the data on the passenger and applies the SWRL rule 4 of [Section 4.2]. Based on the values, the system provided Maria with a seat on the bus and recommended that she take care of her health by taking her medication during the trip. The results are stored as travel history in Maria's passenger profile.

\section{Implementation and Experimentation}

\subsection{Implementation}

The proposed system is implemented with java; it consists of a set of modules, namely: Protégé 2000 to build the ontology, Jena for reasoning, a persistent storage layer, and a SPARQL server. RDF files containing all passenger information are used in the system. The files are generated after the connection has been established between the smart bus station and the person who requested the transport. The file consists of personal information, medical profile, previous journeys, data collected from wearable devices, etc. We have built a system that simulates the smart bus stop. It is designed as a dashboard to show and explain the different steps taken to process the data stored in the 
passengers' RDF files. Once the data processing operation is done, the system displays the results represented as a set of services adapted to each passenger's situation.

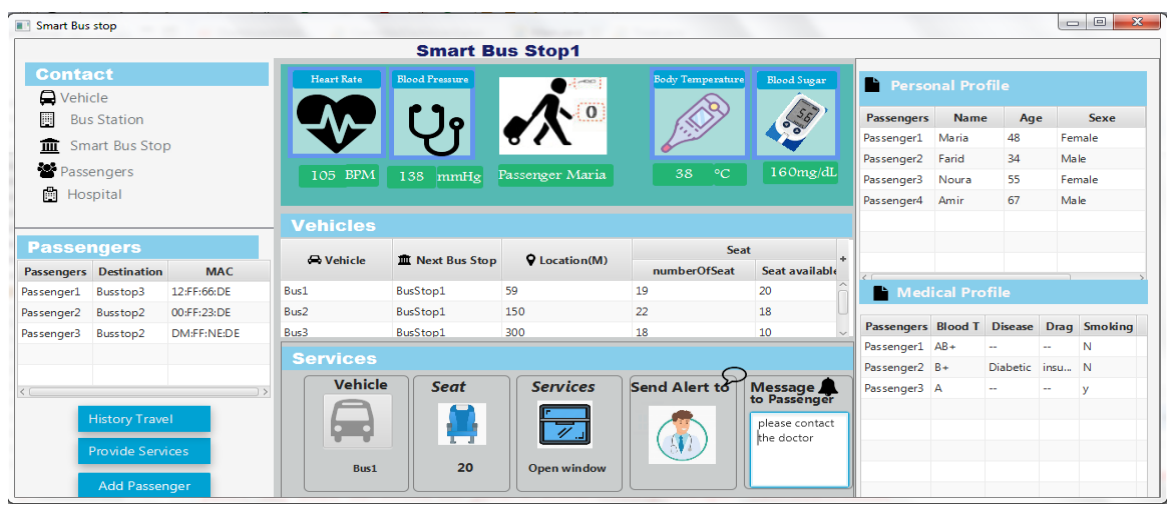

Figure 6: Dashboard of our system

The dashboard shown in Figure 6 uses the SPARQL query to retrieve information from the RDF file for processing and display on its panels. It contains a set of access points presented by buttons that allow us to collect the exchanged data. The "Contact" panel shows all possible contacts made by the smart bus stop to collect the required data from passengers and vehicles. The panel shows the exchange of information with other smart bus stops, bus stations and the transport agency, and messages that inform passengers, drivers, physicians, etc. In the "Passengers" panel, we present data on passengers, such as their identification, MAC address, and destinations. We implemented the Maria passenger scenario mentioned in [section 5] by reusing the metrics presented in the system health metrics panel. We also used the data displayed in the "Vehicles" panel, which shows the vehicles located near the smart bus stop, the number of passengers carried by the vehicles, and the number of seats available for new passengers. The two panels, "Medical Profile" and "Personal Profiles", provide data on the health status of passengers and general information, respectively. This information helps the system to make accurate decisions.

In the example, the passenger has no travel history, so the system stores future outcomes in a history file for the passenger. The system makes decisions based on a set of services and recommendations through the data displayed in the previous panels. The "services" panel shows that the passenger Maria, depending on her health condition, will benefit from the "bus" type vehicle (Bus 1), seat number 20, and with an open window. In addition, a message was sent to inform her physician of her health condition, and another message was sent to Maria to ask her to take medication or to contact her physician.

\subsection{Experiment}

Tests were carried out on the biometric data of a group of people to evaluate the performance of the proposed approach. At the same time, we asked a medical specialist to read the same data and give suggestions according to each person's medical 
condition. Finally, we compared the recommendations of the system with those of the physician.

\subsubsection{Data collection}

A database of vital signs from 18 volunteers provided by the Figshare ${ }^{5}$ repository was used to test the system. To overcome the lack of biometric data that could cover as many cases as possible, the dataset presented in Table 2 was enriched with the help of a physician by proposing new metrics representing critical situations that passengers might encounter in addition to medical profile information such as gender, age, etc.

\begin{tabular}{|c|c|c|c|c|c|c|c|c|c|c|c|c|}
\hline $\begin{array}{l}0 \\
0 \\
0 \\
0 \\
0 \\
0 \\
0 \\
0 \\
0 \\
0\end{array}$ & 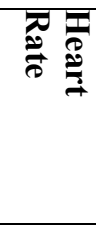 & 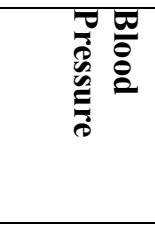 & 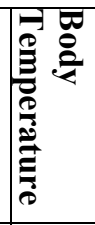 & $\underset{\tilde{D}}{\tilde{N}}$ & 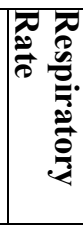 & $\underset{\sigma}{\overrightarrow{\sigma o g}}$ & 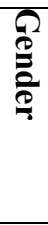 & 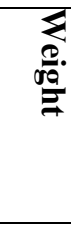 & 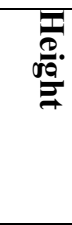 & & & \\
\hline P1 & 98 & $109 / 73$ & 37 & 96 & 15 & 22 & 1 & 61 & 64 & 1 & 1 & 1 \\
\hline $\mathrm{P} 2$ & 193 & $140 / 90$ & 36,7 & 98 & 11 & 38 & 1 & 119 & 171 & 1 & 1 & 2 \\
\hline P3 & 91 & $106 / 68$ & 36,5 & 97 & 18 & 26 & 1 & 113 & 177 & 1 & 1 & 1 \\
\hline P4 & 98 & $109 / 73$ & 36,3 & 99 & 18 & 19 & 2 & 58 & 161 & 1 & 1 & 1 \\
\hline P5 & 99 & $90 / 60$ & 37,2 & 97 & 12 & 20 & 2 & 60 & 168 & 1 & 1 & 1 \\
\hline P6 & 80 & $112 / 72$ & 36 & 97 & 29 & 20 & 2 & 65 & 151 & 1 & 1 & 2 \\
\hline P7 & 103 & $122 / 78$ & 37,2 & 99 & 20 & 28 & 1 & 72 & 164 & 1 & 2 & 1 \\
\hline P8 & 73 & $104 / 67$ & 36,8 & 98 & 26 & 75 & 2 & 51 & 157 & 1 & 2 & 1 \\
\hline P9 & 72 & $180 / 97$ & 39,3 & 94.3 & 20 & 80 & 1 & 98 & 166 & 1 & 1 & 1 \\
\hline P10 & 116 & $190 / 117$ & 38,7 & 95.2 & 22 & 85 & 2 & 86 & 160 & 2 & 1 & 2 \\
\hline P11 & 81 & $107 / 64$ & 37,1 & 77 & 14 & 37 & 2 & 64 & 160 & 1 & 1 & 1 \\
\hline P12 & 90 & $140 / 90$ & 40,7 & 98 & 24 & 43 & 1 & 119 & 173 & 1 & 1 & 1 \\
\hline P13 & 97 & $114 / 62$ & 36,8 & 97 & 16 & 20 & 2 & 55 & 145 & 1 & 2 & 1 \\
\hline P14 & 92 & $99 / 63$ & 39,3 & 89 & 10 & 26 & 2 & 74 & 164 & 1 & 2 & 1 \\
\hline P15 & 80 & $126 / 78$ & 37,2 & 97 & 20 & 67 & 1 & 65 & 166 & 2 & 2 & 1 \\
\hline P16 & 103 & $113 / 73$ & 36,4 & 98 & 20 & 20 & 1 & 94 & 170 & 2 & 1 & 2 \\
\hline P17 & 100 & $152 / 90$ & 38,2 & 99 & 22 & 55 & 2 & 61 & 167 & 1 & 1 & 1 \\
\hline P18 & 81 & $104 / 68$ & 34,8 & 98 & 16 & 26 & 1 & 74 & 176 & 1 & 1 & 1 \\
\hline P19 & 98 & $159 / 97$ & 35,5 & 96 & 25 & 23 & 1 & 78 & 182 & 1 & 1 & 1 \\
\hline $\mathrm{P} 20$ & 193 & $117 / 62$ & 38,4 & 96 & 23 & 24 & 1 & 103 & 184 & 2 & 1 & 1 \\
\hline $\mathrm{P} 21$ & 98 & $100 / 61$ & 40,1 & 94.4 & 28 & 66 & 2 & 62 & 148 & 2 & 2 & 1 \\
\hline $\mathrm{P} 22$ & 81 & $81 / 55$ & 36,9 & 99 & 15 & 28 & 1 & 55 & 142 & 2 & 1 & 1 \\
\hline P23 & 91 & $110 / 61$ & 38,9 & 94.5 & 24 & 30 & 2 & 75 & 136 & 2 & 1 & 1 \\
\hline P24 & 98 & $125 / 66$ & 39,3 & 95.4 & 20 & 35 & 1 & 60 & 130 & 1 & 2 & 1 \\
\hline $\mathrm{P} 25$ & 109 & $132 / 61$ & 40,7 & 94.4 & 21 & 40 & 2 & 65 & 124 & 1 & 1 & 1 \\
\hline
\end{tabular}

${ }^{[5]}$ https://figshare.com/articles/dataset/Vital_signs_of_15_Volunteers/5562298/1 


\begin{tabular}{|l|l|l|l|l|l|l|l|l|l|l|l|l|}
\hline P26 & 81 & $139 / 86$ & 38,1 & 95.3 & 12 & 45 & 1 & 80 & 118 & 1 & 1 & 2 \\
\hline P27 & 103 & $137 / 90$ & 34.5 & 94.5 & 23 & 50 & 1 & 74 & 112 & 1 & 1 & 1 \\
\hline P28 & 77 & $95 / 86$ & 37.1 & 97.1 & 10 & 55 & 2 & 58 & 106 & 1 & 1 & 1 \\
\hline P29 & 72 & $119 / 77$ & 36 & 96 & 19 & 60 & 1 & 72 & 100 & 1 & 1 & 1 \\
\hline P30 & 113 & $160 / 99$ & 39.5 & 97.2 & 11 & 65 & 1 & 63 & 119 & 1 & 2 & 2 \\
\hline
\end{tabular}

Gender $=1$ indicates that the patient is male; 2 indicates that the patient is female.

Alcohol $=1$ indicates that the patient drinks alcohol; 2 indicates that the patient does not drink.

Smoking $=1$ indicates that the patient smokes, 2 indicates that the patient does not smoke.

Medication=1 indicates that the patient has medication, 2 indicates that the passenger has no medication.

\section{Table 2: The dataset used for the test}

\subsubsection{Methodology of Experimentation}

The experiments were conducted under the supervision of Dr. A. KIRATI, a specialist in cardiovascular diseases from the city of Guelma in Algeria, to evaluate the performance of the proposed approach. Before the experimentation, the physician checked the dataset; she was very satisfied with its richness, as she stated that it covers as many cases as possible. The dataset is provided to the physician with a questionnaire that defines the type of vehicle that should be recommended and other services such as air-conditioning, drinks, etc. We asked the physician to mention who should be alerted by a message, such as the hospital, passengers or doctors. After studying and analysing the passengers' data, the physician answered the questionnaire; then, we compared the physician's answers with the result of the proposed system to evaluate its performance.

\subsubsection{Result of Experimentation}

The result of the questionnaire presented in Figure 7 shows that the physician recommended to $30 \%$ of the 30 passengers to take a bus (service 1 ), to $3.3 \%$ to use a bus with other services such as an open window (service 2 ) and to $16.6 \%$ to use a car to travel and contact doctors (service 3 ). The physician recommended to $50 \%$ to be assisted by an ambulance in case of emergency (service 4). The result of the proposed system for the same dataset of 30 passengers was as follows: it recommended to $30 \%$ to take a bus (service 1), to $6 \%$ the bus with other services (service 2), and to $24 \%$ a car with contact to doctors (service 3 ) and to $33 \%$ an ambulance in case of emergency (service 4). 

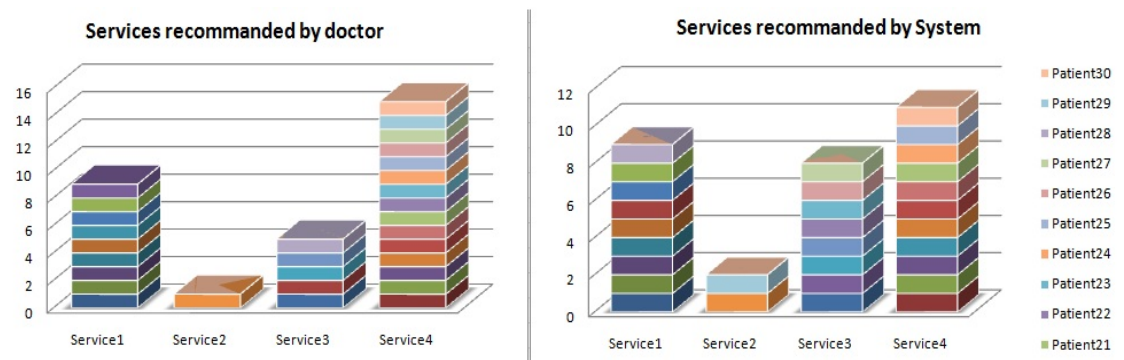

Figure 7: Comparison between the physician referral service and the proposed system

The comparison between the two results of the experiment and the approach can be divided into three categories, as shown in Figure 8 . The first one shows the conformity of both results at $70 \%$, and the second one shows the convergence of the services provided without risk to the health of the passengers at $27 \% ; 3 \%$ shows a divergence.

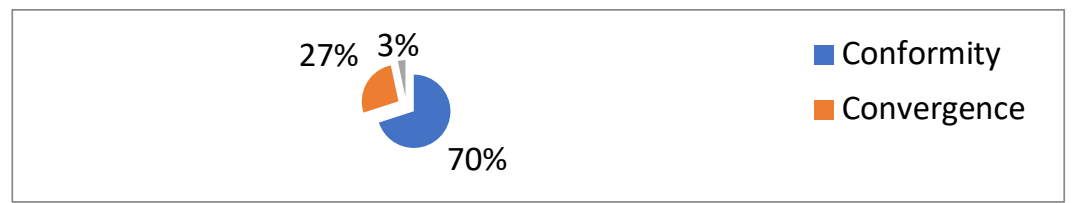

Figure 8: Similarity of results

\subsubsection{Discussion}

The experiment showed the high rate of coincidence of results between the proposed approach and the physician's recommendation (70\%), which proves its competence to provide consistent services through a level of reasoning as high as the physician's to reach accurate decisions for heterogeneous sensor data collected in different domains. The $27 \%$ convergence means that the system needs to add additional capabilities to handle the imprecision of the range of vital signals. The erroneous recommendations with a rate of $3 \%$ have many explanations (errors in the value of the measurements, error in the SWRL rules, etc.), which can be confirmed by applying more experimentation. The performance of the system presents its capabilities to achieve semantic interoperability. Ultimately, it is about exchanging the heterogeneous IoT data created from two domains and allowing them to derive new knowledge that could help predict the health of passengers. Physicians or hospitals can use the system in health surveillance of epidemics, such as Covid-19, or it can be used in analytical studies of disease spread patterns. 


\section{Conclusion}

This paper proposed a system based on a Semantic Web of Things to manage the integration of public transport and health monitoring challenges to provide high-quality transport services and ensure continuous health monitoring. The system focuses on the Internet of Things at the edge, fog and cloud level to collect data, exchange it with different resources and process it to make decisions and provide health services and recommendations. The system architecture is based on the smart bus stop, which functions as an edge, and some interconnected bus stations constitute a fog, while the transport agency functions as a cloud. The proposed approach uses the Semantic Web to overcome the heterogeneity of the devices' data and the two domains (public transport and health).

The approach is based on an ontology that presents relevant concepts related to passenger health and transport management. In addition, SWRL rules are used to analyse passenger profiles based on data sent by wearable devices. Inference engines and query engines are used to provide appropriate services and manage the interaction with the application. The proposed ontology has been designed in three steps. The first step is to create a new ontology that presents the appropriate public transport with the proposed architecture. In the second step, we reused the extension of the SAREF ontology for wearable devices. In the third step, we merge the two ontologies with some modifications to create a new ontology. The implementation and experimentation demonstrated that the proposed system allows achieving semantic interoperability across a cross-domain.

In future work, we will focus on the pre-processing phase to improve the quality of the data, avoid the faulty diagnostic state, detect missing data and eliminate spurious data. We will choose to use machine learning techniques to improve the data analysis and processing performance. In addition, we will extend the system to address security challenges, mainly because the devices in the system are highly dispersed and exchange critical data.

\section{References}

[Alaya et al., 2015] Alaya, M. B., Medjiah, S., Monteil, T., Drira, K.: "Toward semantic interoperability in oneM2M architecture"; IEEE Communications Magazine, 53(12), (2015), 3541. doi: 10.1109/MCOM.2015.7355582.

[Ashton, 2009] Ashton, K.: "That'internet of things' thing"; RFID journal, 22(7), (2009), 97-114.

[Avancha et al., 2004] Avancha, S., Patel, C., Joshi, A.: "Ontology-driven adaptive sensor networks"; UMBC Student Collection, (2004). doi : 10.1109/MOBIQ.2004.1331726.

[Benvenuti et al., 2017] Benvenuti, F., Diamantini, C., Potena, D., Storti, E.:"An ontology-based framework to support performance monitoring in public transport systems"; Transportation Research Part C: Emerging Technologies, 81, (2017), 188-208.doi: 10.1016/j.trc.2017.06.001.

[Bermejo et al., 2014] Bermejo, A. J., Villadangos, J., Astrain, J. J., Córdoba, A., Azpilicueta, L., Garate, U., Falcone, F.: "Ontology Based Road Traffic Management in Emergency Situations";Adhoc\& Sensor Wireless Networks, 20, (2014),47-69. 
[Bröring et al., 2017] Bröring, A., Schmid, S., Schindhelm, C. K., Khelil, A., Käbisch, S., Kramer, D., Teniente, E.: Enabling IoT ecosystems through platform interoperability; IEEE software, 34(1), (2017), 54-61. doi: 10.1109/MS.2017.2.

[Compton et al., 2012] Compton, M., Barnaghi, P., Bermudez, L., GarcíA-Castro, R., Corcho, O., Cox, S., Taylor, K.: "The SSN ontology of the W3C semantic sensor network incubator group"; Journal of Web Semantics, 17, (2012), 25-32. doi: 10.1016/j.websem.2012.05.003.

[Daniele et al., 2015] Daniele, L., den Hartog, F., Roes, J.: "Created in close interaction with the industry: the smart appliances reference (SAREF) ontology"; In International Workshop Formal Ontologies Meet Industries. Springer, Cham, (2015), 100-112. https://doi.org/10.1007/978-3319-21545-7_9.

[El-Sappagh et al., 2019] El-Sappagh, S., Ali, F., Hendawi, A., Jang, J. H., Kwak, K. S.: "A mobile health monitoring-and-treatment system based on integration of the SSN sensor ontology and the HL7 FHIR standard"; BMC medical informatics and decision making", 19(1),(2019), 97. https://doi.org/10.1186/s12911-019-0806-z.

[Faheem et al., 2019] Faheem, M. R., Anees, T., Hussain, M.:" The Web of things: Findability taxonomy and challenges"; IEEE Access, 7 (2019), 185028-185041. doi: 10.1109/ ACCESS. 2019.2960446.

[Frez et al., 2019] Frez, J., Baloian, N., PINO, J., et al.: "Planning of urban public transportation networks in a Smart City"; (JUCS-Journal of Universal Computer Science),25, (2019),946-966. doi: 10.3217/jucs-025-08-0946.

[Ganzha et al., 2017] Ganzha, M., Paprzycki, M., Pawłowski, W., Szmeja, P., Wasielewska, K.:"Semantic interoperability in the Internet of Things: An overview from the INTER-IoT perspective"; Journal of Network and Computer Applications, 81, (2017), 111-124. https://doi.org/10.1016/j.jnca.2016.08.007.

[Hitzler, 2021] Hitzler, P.: "A review of the semantic web field"; Communications of the ACM, 64, 2 (2021), 76-83. https://doi.org/10.1145/3397512.

[Houda et al., 2010] Houda, M., Khemaja, M., Oliveira, K., Abed, M.: "A public transportation ontology to support user travel planning"; In 2010 Fourth International Conference on Research Challenges in Information Science (RCIS), IEEE, (2010), 127-136. https://doi.org/10.1109/ RCIS .2010. 5507372.

[Kelly et al., 2020] Kelly, J. T., Campbell, K. L., Gong, E., Scuffham, P.: "The Internet of Things: Impact and implications for health care delivery"; Journal of medical Internet research, 22, 11(2020),1-11. doi:10.2196/20135.

[Manyika et al., 2015] Manyika, J., Chui, M., Bisson, P., Woetzel, J., Dobbs, R., Bughin, J., Aharon, D.: "Unlocking the Potential of the Internet of Things. McKinsey Global Institute", (2015), 1-4.

[Mohan at al., 2016] Mohan, N., Kangasharju, J. Edge-Fog cloud: "A distributed cloud for Internet of Things computations"; In Cloudification of the Internet of Things IEEE, (2016), 1-6. doi: 10.1109/CIOT.2016.7872914.

[Moreira and al., 2020] Moreira, J., Pires, L. F., van Sinderen, M., Daniele, L., Girod-Genet, M.: "SAREF4health: Towards IoT standard-based ontology-driven cardiac e-health systems"; Applied Ontology,15,3, (2020), 385-410. doi: 10.3233/AO-200232.

[Noura et al., 2019] Noura, M., Atiquzzaman, M., Gaedke, M.: "Interoperability in Internet of things: Taxonomies and open challenges"; Mobile Networks and Applications, 24(3), (2019), 796-809. doi: 10.1007/s11036-018-1089-9. 
[Reda et al., 2018] Reda, R., Piccinini, F.,Carbonaro, A.: "Towards consistent data representation in the IoT healthcare landscape"; In Proceedings of the 2018 International Conference on Digital Health, April 2018, 5-10. doi: 10.1145/3194658.3194668.

[Rhayem et al., 2017] Rhayem, A., Mhiri, M. B. A., Gargouri, F.: "HealthIoT ontology for data semantic representation and interpretation obtained from connected medical objects"; 14 th International Conference on Computer Systems and Application. IEEE, (2017), 1470-1477. doi: 10.1109/AICCSA.2017.171.

[Ryu et al., 2020] Ryu, S., Park, B. B., El-Tawab, S.: "WiFi Sensing System for Monitoring Public Transportation Ridership: A Case Study"; KSCE Journal of Civil Engineering, 24(10), (2020), 3092-3104. doi: 10.1007/s12205-020-0316-7.

[Scioscia and Ruta, 2009] Scioscia, F., Ruta, M.: "Building a Semantic Web of Things: issues and perspectives in information compression"; In 2009 IEEE International Conference on Semantic Computing. IEEE, (2009), 589-594. doi: 10.1109/ICSC.2009.75.

[Suárez-Figueroa et al., 2015] Suárez-Figueroa, M. C., Gómez-Pérez, A., Fernandez-Lopez, M.:"The NeOn Methodology framework: A scenario-based methodology for ontology development"; Applied ontology; 10, 2(2015), 107-145. doi: 10.3233/AO-150145.

[Studer et al., 1998] Studer, R., Benjamins, V. R., Fensel, D.: "Knowledge engineering: Principles and methods"; Data \& knowledge engineering, 25(1-2), (1998), 161-197. doi: 161197. 10.1016/S0169-023X(97)00056-6.

[Takei et al., 2015] Takei, K., Honda, W., Harada, S., Arie, T., Akita, S.: "Toward flexible and wearable human interactive health monitoring devices". Advanced healthcare materials, 4(4), (2015), 487-500. doi: 10.1002/adhm.201400546. 